\title{
Applications of simple robots to encourage social receptiveness of adolescents with autism
}

\author{
Sandra Costa, Jorge Resende, Filomena Oliveira Soares, Manuel João Ferreira, Cristina P. Santos, \\ Fátima Moreira
}

\begin{abstract}
This paper reports the preliminary research studies regarding the use of LEGO Mindstorms TM robots as a therapy for autistic young people. Most autistic people hardly communicate in a social environment, living in their own world. This is the first step in a broader project which aims to improve the autistic people social life and also their ability to communicate, using the robot as a social bridge between them and the surrounding environment/people.

The target group is 17-19 years old having autism disorder and developmental disability. The trials consists of undemanding plays where the intervention of the autistic person is required, using simple inputs, such as voice commands, pressing a button or clapping hands. The presented work intends to evaluate the reaction of this particular target group to the introduction of a toy-like robot in their classroom daily life. The methodology applied, the detailed description as well as the analysis of the experiments are presented and discussed.
\end{abstract}

\section{INTRODUCTION}

A utism is a complex developmental disability that typically appears during the first three years of life. It results from neurological disorders that affect the functioning of the brain and impact the brain normal development mainly in the areas of social interaction and communication skills. Children and adults with autism typically have difficulties in verbal and non-verbal communication, social interactions, and leisure or play activities [1]. Autism symptoms and characteristics appear in a wide variety of combinations, from mild to severe. But usually these individuals show impairments in social interaction, communication, imagination (repetitive behaviour) and fantasy.

There are a few projects related to this theme [2] - [6]. Among them special focus will be given to IROMEC, ROBOTA and AURORA projects.

The project AURORA has been investigating the use of a robotic platform as a tool for therapy with children with autism [2]. A key issue in this project is the evaluation of the

Sandra Costa, Jorge Resende Filomena Oliveira Soares, Manuel João Ferreira (corresponding author phone: +351253510190; fax: +351253510189; email: mjf@dei.uminho.pt) and Cristina P. Santos are with the Industrial Electronics Department, University of Minho, Guimarães, Portugal.

Fátima Moreira is with the APPACDM (an association for mental disable people; in Portuguese: Associação de Pais e Amigos do Cidadão com Deficiência Mental), Braga, Portugal. interactions, which are not constricted and involve the child to move without restraint in the class.

The ROBOTA project is part of a current trend of robotics research that develops educational robot toys. ROBOTA is the name of a series of doll-shaped mini-humanoid robots, whose physical features resemble those of a human baby. These robots have been applied as assistive technologies in behavioural studies of low-functioning children with autism. These studies investigate the potential of using an imitator robot to assess children's imitation ability and to teach children simple coordinated behaviours [3]. These robots were also used in the AURORA project [2] to verify the effects of repeated exposure of children with autism to a humanoid robot. They concluded that the repeated trials over a long period of time allowed the children to calmly explore the robot-human and human-human interaction.

The project IROMEC proposes to develop a robotic toy for children with cognitive impairments. The project investigates how robotic toys can become social mediators, encouraging children with disabilities to discover a range of play styles, from solitary to social and cooperative play (with peers, carers/teachers, parents). In their results, they present a list of main aspects to take into consideration when designing a (robotic) toy that could assist the play of children with autism, like type of play and movement. [4].

The work described in this paper is part of a research project which aims at applying robotic tools as a mean to improve the social life of adolescents with cognitive impairments, autism disorder and mental disease. In particular, the goal is to improve their interaction and communication abilities with the environment and with other people. In this paper, we report the project first phase in which a specific robot, a simple LEGO robot [7], is introduced in the autistic adolescent classroom. We present and discuss the different trials that were performed, focusing on the evaluation of their reaction to the presence of the robot.

The article is divided in four sections. First, a brief introduction of the subject is presented, highlighting the work developed by other research teams. The methodology applied as well as a detailed description and the analyses of the experiments are fully explained in section 2 . Results are presented in section 3. Finally, the conclusions and the future work are listed. 


\section{METHODOLOGY}

In this section the methodology employed in the research work is detailed. It is presented the organizational environmental and the session settings, focusing on the classroom setup and the robot configuration. The target group is characterized and the sessions are explained.

\section{A. The organizational environment}

This research work is a partnership between University of Minho and APPACDM (an association for mental disable people; in Portuguese: Associação de Pais e Amigos do Cidadão com Deficiência Mental). The main goal of APPACDM is to promote the integration, in the society, of the citizens with mental deficiency, as well to guarantee the emotional equilibrium of the respective families.

\section{B. The session's settings}

The different experiences took place in the facilities of the APPACDM in Gualtar (figure 1). The addressed target group is very sensitive to changes in their routine daily life, namely to changes in their usual surrounding environment. In order to reduce the stress associated with these changes, experiments were performed in the classroom where the adolescents usually exercise. In this room there are seven adolescents with mental impairments; two with both mental impairment and autism and two carers. To each adolescent has been delineated a specific educational program. The classroom has been equipped with video cameras so that both the classroom daily life and the sessions could be recorded for analysis.

\section{The robot}

The robot used in the experiences is a LEGO MindStorms NTX (figure 2). At this stage we have adopted a non humanlike shape.

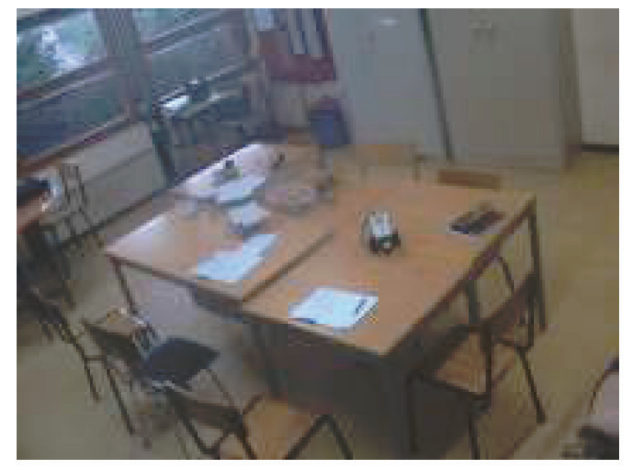

Fig. 1. Classroom environment during trial

The robot was programmed for two distinct experiences:

- Trial 1 - This experiment is designed to address the adolescent reaction to sound. The robot executes a predefined choreography only when its touch sensor is pressed. This choreography consists of a robot movement from back to front and backwards robot movement, followed by a circular robot movement, while moving its end-effector.

- Trial 2, reaction to sound - In this trial the goal is to address the robot reaction to sound. Once a certain sound (music, clapping, among others) is higher than a predefined value, the robot performs a similar choreography.

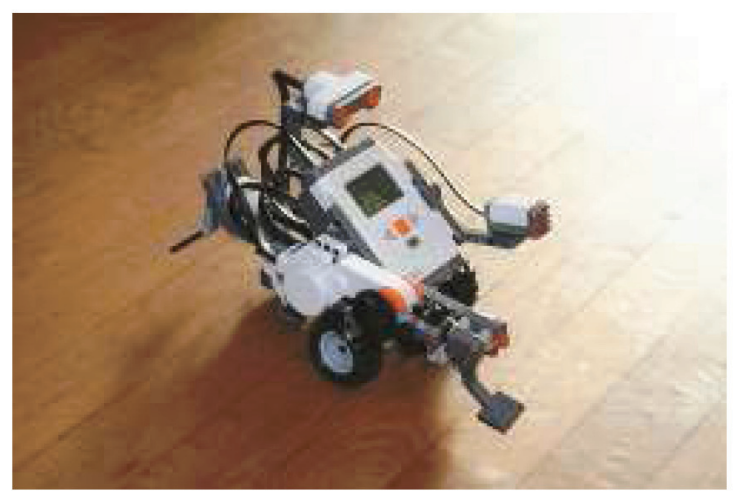

Fig. 2. Robot Configuration

\section{The adolescents}

Two autistic adolescents (17-19 years old) with mental disorders were chosen as the target group. These adolescents have autistic characteristics somewhat different from each other. Adolescent 1 does not like the changes in his daily routine and these changes can cause an aggressive behavior. On the other hand, adolescent 2 presents a less aggressive behavior and does not require so many cares. Both have some difficulties in communication, especially in speaking.

\section{E. The sessions}

The experimental sessions were specified, by the researchers and the carers, according to these common characteristics of the adolescents, such as difficulty in developing social relationships and communications. Carers are the persons that best know each of these adolescents and their help was relevant to the experiment specification and interpretation.

Three basic stages were defined: exploration, demonstration and interaction phases.

In the exploratory phase the robot was presented gradually by the carer to the adolescent, allowing them to observe and accept it as an object in their routine. In the demonstration phase, the adolescent was accompanied by the carer, the researchers and the robot.

In this second phase, the control of the experiment was performed by the researcher. This was one of the test parameters in order to to understand how the target group accept orders/commands from the investigator. The researcher showed an activity of interaction with the robot, presenting some of its features, allowing the adolescent to 
have a new form of interaction and integration. This experience was repeated five times with each autistic in order to find any kind of pattern behavior in the adolescents.

In the interaction phase, to be performed in the near future, the adolescent will be encouraged to interact with the robot, developing their capacities of communication.

\section{RESULTS}

During the demonstration phase five sessions with each adolescent were performed. These sessions were planned in such a way that all of them have the same time of duration and the same operation modes, allowing to compare results and to verify the evolution in the robot-subject interaction.

The planned sessions produced different results. Note that for Adolescent 2 only 4 sessions were performed.

Adolescent 1 preferred to activate the robot through the touch sensor, while Adolescent 2 preferred the sound sensor, but throughout the sessions there have been evolution in both, denoted by the increase in the number of occurrences (Figs. 3 to 6).

Adolescent 2 showed some interest untill the $3^{\text {rd }}$ session, but on the others showed little interest in the robot, being necessary to stimulate his participation.

Adolescent 1 improved in the interaction, both in the touch sensor and in the sound sensor. However, the graph in fig. 3 clearly shows how the Adolescent 1 prefers the touch sensor while the Adolescent 2 prefers the sound sensor to activate the robot.

Concerning the reactions to robot, results indicate a more complex pattern for adolescent 2. He showed more disinterest than adolescent 1 in the ongoing of the sessions. In sessions 4 and 5 , adolescent 1 always paid attention to the robot, contrarily to adolescent 2, who often ignored it.

The behaviour regarding fixing in robot's details were similar in both of the adolescents.

Adolescent 1 just explored the robot (robot manipulation) whenever he was not interested in activating it. In these cases, he looked for our help; if we refused to help him, he started to touch the control buttons with some aggressiveness. In general, adolescent 2 did not look for help, he explored the robot or looked for something to occupy himself.

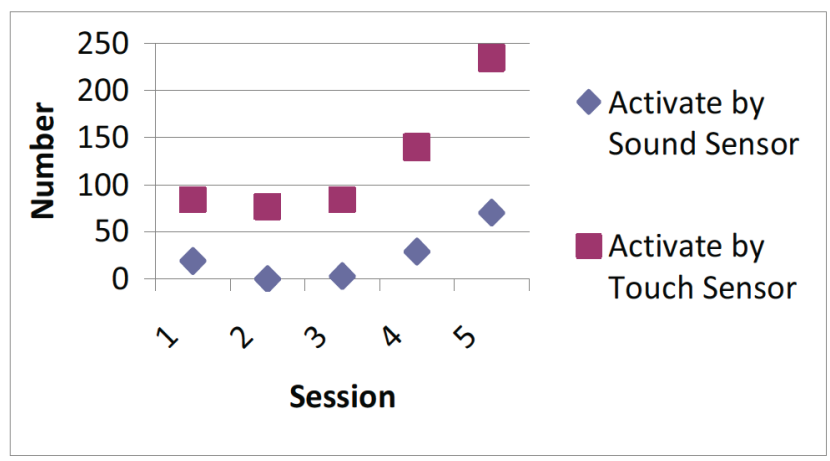

Fig. 3. Number of occurrences for the activation mode of the robot by Adolescent 1.

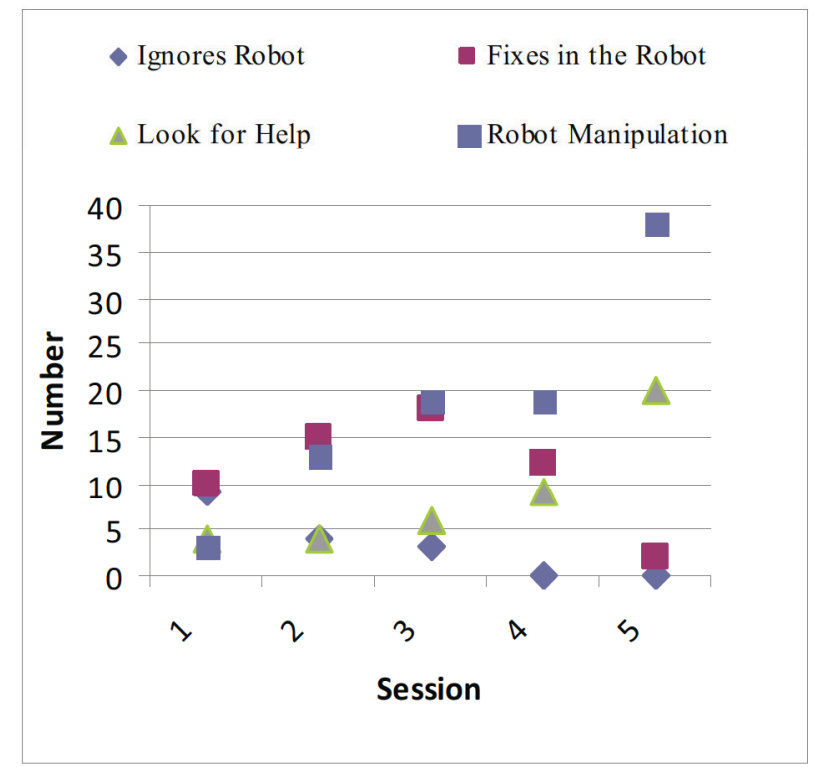

Fig. 4. Number of occurrences of the reactions parameters for the Adolescent 1.

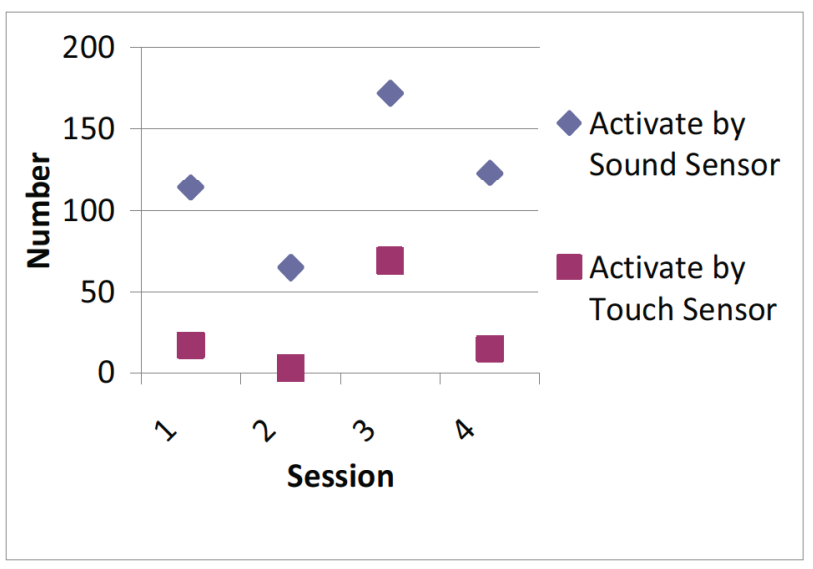

Fig. 5. Number of occurrences for the activation mode of the robot by Adolescent 2.

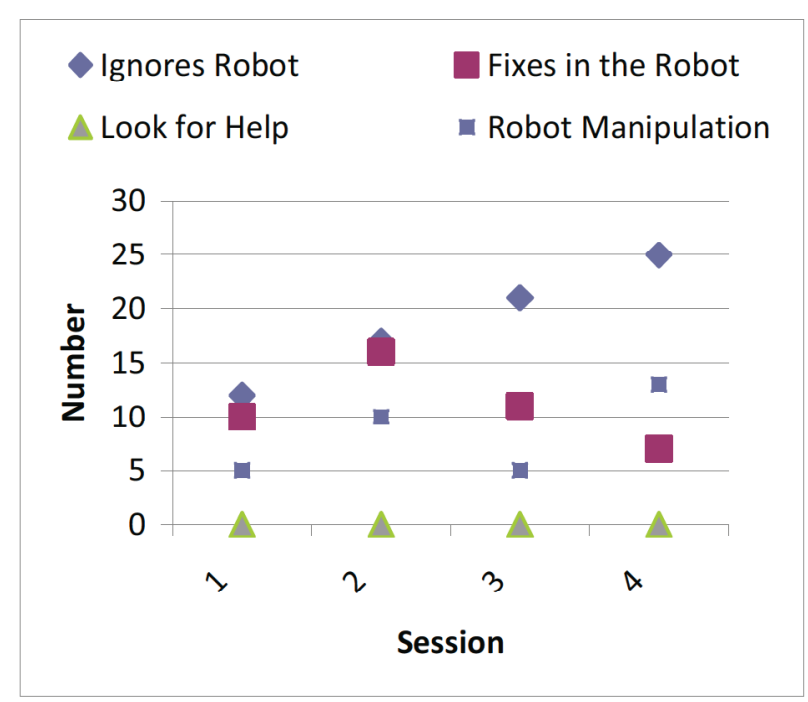

Fig. 6. Number of occurrences of the reactions parameters for the Adolescent 2. 
In relation to the robot's retreat, adolescent 2 revealed indifference, while Adolescent 1 showed an increase in interest for interacting with the robot.

\section{CONCLUSIONS}

The five sessions performed allowed to evaluate the reactions of the two adolescents in the presence of the robot. The following issues can be summarized:

(1) - Interaction was evaluated through the measurement of the number of occurrences of: robot's activation by sound sensor and by touch sensor, ignores robot, fixes in the robot, looks for help and robot manipulation;

(2) - both adolescents interacted differently with the robot, especially concerning the robot activation and the robot manipulation;

(3) - Adolescents behave differently concerning the interest in maintaining the interaction throughout time.

The work presented in this article is part of a research project concerning the use of robotic platforms to reach autistic people. In further work, especial attention will be devoted to the temporal analysis, performing a statistical analysis, in order to better understand the evolution of interaction with time. Additionally, other robot configurations will be tested to evaluate the relation between the type of robot and the interaction. It is also our aim to extend this research to more autistic people.

\section{ACKNOWLEDGMENT}

The authors are grateful to the teaching staff, parents and adolescent at APPACDM for their collaboration and partnership.

\section{REFERENCES}

[1] E. Sutinen, M. Virmajoki-Tyrväinen and M. Virnes, Concretizing Technologies in Special Education for Developing Social Skills, in www-edc.eng.cam.ac.uk/cwuaat/04/48-pat-cmc-

virnes_specedu_final.pdf (accessed on January 2009)

[2] Kerstin Dautenhahn (1999), Robots as Social Actors: AURORA and the Case of Autism, Proceedings CT99, The Third International Cognitive Technology Conference, August 1999, San Francisco, USA

[3] A. Billard, B. Robins, K. Dautenhahn and J. Nadel, Building Robota, a Mini-Humanoid Robot for the Rehabilitation of Children with Autism, the RESNA Assistive Technology Journal, 19 (2006).

[4] Robins, B., Otero, N., Ferrari, E. and Dautenhahn, K. (2007) ' Eliciting Requirements for a Robotic Toy for Children with Autism Results from User Panels.' Procs 16th IEEE Int Symp on Robot and Human Interactive Communication (RO-MAN2007).

[5] B. Robins, K. Dautenhahn, R. te-Boekhorst, and A. Billard, "Robotic assistants in therapy and education of children with autism: can a small humanoid robot help encourage social interaction skills?," Universal Access in the Information Society, vol. 4:2. , 2005.

[6] H. Kozima, M.P. Michalowski, C. Nakagawa, Keepon: A Playful Robot for Research, Therapy, and Entertainment, International Journal of Social Robotics, Vol. 1, 2008.

[7] http://mindstorms.lego.com/eng/Antarctica_dest/Default.aspx (accessed on January 2009) 